\title{
Maternal chromium restriction induces insulin resistance in adult mice offspring through miRNA
}

\author{
QIAN ZHANG ${ }^{1}$, XIAOFANG SUN $^{2}$, XINHUA XIAO $^{1}$, JIA ZHENG $^{1}$, MING LI $^{1}$, MIAO YU $^{1}$, FAN PING $^{1}$, \\ ZHIXIN WANG ${ }^{1}$, CUIJUAN QI ${ }^{1}$, TONG WANG ${ }^{1}$ and XIAOJING WANG ${ }^{1}$ \\ ${ }^{1}$ Key Laboratory of Endocrinology, Translational Medicine Centre, Ministry of Health, Department of Endocrinology, \\ Peking Union Medical College Hospital, Peking Union Medical College, Chinese Academy of Medical Sciences, \\ Beijing 100730; ${ }^{2}$ Department of Endocrinology, The Affiliated Hospital of Qingdao University, \\ Qingdao, Shandong 266003, P.R. China
}

Received November 10, 2016; Accepted December 8, 2017

DOI: $10.3892 / \mathrm{ijmm} .2017 .3328$

\begin{abstract}
Increasing evidence suggests that undernutrition during the fetal period may lead to glucose intolerance, impair the insulin response and induce insulin resistance (IR). Considering the importance of chromium $(\mathrm{Cr})$ in maintaining carbohydrate metabolism, the present study aimed to determine the effects of maternal low $\mathrm{Cr}$ (LC) on glucose metabolism in C57BL mice offspring, and the involved mechanisms. Weaned C57BL mice were born from mothers fed a control diet or LC diet, and were then fed a control or LC diet for 13 weeks. Subsequently, the liver microRNA (miRNA/miR) expression profile was analyzed by miRNA array analysis. A maternal LC diet increased fasting serum glucose $(\mathrm{P}<0.05)$ and insulin levels $(\mathrm{P}<0.05)$, homeostasis model assessment of IR index $(\mathrm{P}<0.01)$, and the area under curve for glucose concentration during oral glucose tolerance test $(\mathrm{P}<0.01)$. In addition, 8 upregulated and 6 downregulated miRNAs were identified in the maternal LC
\end{abstract}

Correspondence to: Professor Xinhua Xiao, Key Laboratory of Endocrinology, Translational Medicine Centre, Ministry of Health, Department of Endocrinology, Peking Union Medical College Hospital, Peking Union Medical College, Chinese Academy of Medical Sciences, 1 Shuaifuyuan Road, Dongcheng District, Beijing 100730, P.R. China E-mail: xiaoxh2014@vip.163.com

Abbreviations: Akt1, thymoma viral proto-oncogene 1; AUC, area under the curve; $\mathrm{CON}$, control; $\mathrm{Cr}$, chromium; GK, Goto-Kakizaki; Glut4, glucose transporter 4; GO, Gene Ontology; HOMA-IR, homeostasis model assessment of insulin resistance; IR, insulin resistance; IRS1, insulin receptor substrate 1; KEGG, Kyoto Encyclopedia of Genes and Genomes; LC, low chromium; miRNA, microRNA; NC, negative control; OGTT, oral glucose tolerance test; Pdpk1, 3-phosphoinositide dependent protein kinase 1; Pik3ca, phosphatidylinositol 3-kinase, catalytic, $\alpha$ polypeptide; Pik3r1, phosphatidylinositol 3-kinase, regulatory subunit, polypeptide; Pik3r3, phosphatidylinositol 3-kinase, regulatory subunit, polypeptide 3 ; $\mathrm{PKB}$, protein kinase $\mathrm{B}$; Slc2a4, solute carrier family 2 member 4 ; T2D, type 2 diabetes

Key words: fetal programming, epigenetics, maternal environment, chromium, microRNA group (fold change $\geq 2, \mathrm{P}<0.05$ ). miRNA-gene networks, Kyoto Encyclopedia of Genes and Genomes pathway analysis of differentially expressed miRNAs, and miRNA overexpression in HepG2 cells revealed the critical role of insulin signaling, via miR-327, miR-466f-3p and miR-223-3p, in the effects of early life $\mathrm{Cr}$ restriction on glucose metabolism. In conclusion, maternal $\mathrm{Cr}$ restriction may irreversibly increase IR, which may involve a specific miRNA affecting the insulin signaling pathway.

\section{Introduction}

An increasing number of individuals are being diagnosed with diabetes, particularly type 2 diabetes (T2D) (1). In addition to genetic factors, environmental factors contribute to the occurrence of T2D. In human and animal experiments, a negative association between intrauterine nutrition and metabolic disease risk has been observed. Hales et al reported a U-shaped relationship between birth weight and glucose intolerance at 64 years of age (2). Furthermore, low-birth weight subjects exhibited increased plasma levels of proinsulin, which is an indication of islet $\beta$ cell dysfunction. Based on these findings, Hales and Barker proposed the 'thrifty phenotype hypothesis', which addresses the important role of poor nutrition in early life on permanent changes in glucose-insulin metabolism, to explain this relationship (3).

Chromium $(\mathrm{Cr})$ is an essential glucose regulator $(4,5)$. The normal human range of blood $\mathrm{Cr}$ is $0.12-0.67 \mu \mathrm{g} / 1$ (6). However, total body $\mathrm{Cr}$ concentrations decrease by $25-40 \%$ with age. Subjects with $\mathrm{Cr}$ deficiency have increased blood glucose and insulin levels (7). In addition, the tissue $\mathrm{Cr}$ levels of patients with diabetes are lower compared with in normal control subjects, and a correlation exists between low serum $\mathrm{Cr}$ levels and the occurrence of T2D (8).

A previous study demonstrated that maternal $\mathrm{Cr}$ deficiency leads to insulin resistance (IR) and impaired glucose tolerance in WNIN rat offspring. The underlying mechanism involves increased oxidative stress (9). In addition, the molecular mechanisms underlying fetal programming may be partly associated with epigenetic regulation of the expression of key genes (10). Our recent research indicates that maternal chromium restriction leads the miRNA dysfunction involved with MAPK signaling pathway in the adipose of female offspring (11). 
MicroRNAs (miRNA/miRs) are associated with important epigenetic mechanisms. miRNAs are short noncoding RNA molecules that bind to target genes to regulate various cellular processes. Increasing research has suggested that miRNAs are involved in the incidence and development of diabetes. miR-125a is upregulated in Goto-Kakizaki (GK) rats (T2D rat model) compared with in normal rats (12), and may lead to IR. In addition, increased miR-143 has been detected in the livers of diabetic rats (13); miR-143 downregulates oxysterol-binding protein-related protein 8 and subsequently impairs the ability of insulin to induce the activation of protein kinase B (AKT) signaling, a central node of insulin action to induce glucose metabolism. Upregulation of miR-181a in diabetic livers and hepatocytes decreases sirtuin 1 expression, thus inactivating insulin signaling and glucose metabolism (14). Furthermore, miR-96 and miR-126 directly target the insulin receptor substrate 1 (IRS1) 3'-untranslated region; a reduction in IRS1 is involved in IR under mitochondrial dysfunction in hepatocytes $(15,16)$. Downregulation of miR-200 also impairs AKT/glycogen synthase kinase-mediated glycogenesis in the liver, resulting in hepatic IR (17).

The present study hypothesized that an association exists between maternal low $\mathrm{Cr}$ (LC) status and epigenetic reprogramming of the transcriptome by means of miRNA in offspring. To test this hypothesis, a whole miRNA expression array was performed in mice exposed to maternal $\mathrm{Cr}$ restriction.

\section{Materials and methods}

Animals and protocol. All procedures were performed in strict accordance with the recommendations of the Guide for the Care and Use of Laboratory Animals of the National Institute of Health (18). The present study was approved by the Animal Care Committee of the Peking Union Medical Hospital (Beijing, China; permit no. MC-07-6004), and all efforts were made to minimize suffering. Female $(n=20)$ and male $(n=10)$ C57BL mice (age, 7 weeks; weight, $18.7 \pm 1.8 \mathrm{~g}$ ) were purchased from the Institute of Laboratory Animal Science, Chinese Academy of Medical Sciences and Peking Union Medical College (Beijing, China; SCXK-2013-0107). All mice were maintained in ventilated individual cages at $24 \pm 1^{\circ} \mathrm{C}$ and had access to food and water ad libitum; lights were switched on between 6 a.m. and 6 p.m. . Mating was confirmed by the presence of sperm in a vaginal smear the following morning. Pregnant females $(n=16)$ were randomly divided into two groups: Control group (CON, fed a standard rodent casein-based diet based on the American Institute of Nutrition AIN-93G diet) and LC diet group (fed a LC diet, which lacked only in $\mathrm{Cr}$ ) (n=8/group; Table I). The diet protocol was maintained throughout the gestation and lactation periods. The concentration of $\mathrm{Cr}$ in the CON and LC diets was 1.19 and $0.14 \mathrm{mg} \mathrm{Cr} / \mathrm{kg}^{-1}$ diet, respectively [assessed by atomic absorption spectrometer (TAS986; Beijing Persee General Corporation, Beijing, China)]. All diets were provided by Research Diets, Inc. (New Brunswick, NJ, USA). Following birth of the offspring, the litter size in each cage was randomly adjusted to 6 pups ( 3 males, 3 females, if possible) to ensure equal nutrition until the pups were weaned. Pups were kept under the same housing conditions as the adult mice. After weaning, pups were randomly subdivided into four groups: i) Pregnancy and lactation $\mathrm{CON}$ diet group fed a CON diet after weaning (CON/CON); ii) pregnancy and lactation $\mathrm{CON}$ diet group fed a LC diet after weaning (CON/LC); iii) pregnancy
Table I. Composition of diets ${ }^{\mathrm{a}}$.

\begin{tabular}{lrr}
\hline Ingredient (g or mg/kg diet) & CON & LC \\
\hline Cornstarch, g & 397.5 & 397.5 \\
Casein, g & 200.0 & 200.0 \\
Dextrinized cornstarch, g & 132.0 & 132.0 \\
Sucrose, g & 100.0 & 100.0 \\
Soybean oil, g & 70.0 & 70.0 \\
Fiber, g & 50.0 & 50.0 \\
Mineral mix, g & 35.0 & 35.0 \\
Vitamin mix AIN-93-VX, g & 10.0 & 10.0 \\
L-Cystine, g & 3.0 & 3.0 \\
Choline bitartrate, g & 2.6 & 2.6 \\
Tert-butylhydroquinone, mg & 14.0 & 14.0 \\
Chromium potassium sulfate & 275.0 & 0.0 \\
12H ${ }_{2}$ O (10.42\% Cr), mg & & \\
\end{tabular}

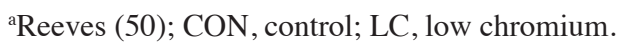

and lactation LC group fed a LC diet after weaning (LC/LC) and iv) pregnancy and lactation LC group fed a CON diet after weaning ( $\mathrm{LC} / \mathrm{CON})$ ( $\mathrm{n}=8 /$ group; 1 male pup from each litter was randomly assigned to the experimental groups). Male offspring only were used for the present study to avoid sex differences on the effect of maternal nutrition on glucose metabolism (19). At the end of the experimental period (16 weeks of age), blood samples were collected from the intraorbital retrobulbar plexus in anesthetized mice (ketamine 100 mg/kg, i.p.; Pfizer, Inc., New York, NY, USA) following a 10 -h fast. All male mice (n=8/group) from eight different litters (1 male offspring/litter) were then sacrificed. Whole livers of the offspring were quickly removed and stored at $-80^{\circ} \mathrm{C}$ for further analysis. The experimental design is presented in Fig. 1.

Measurement of serum Cr levels. The blood samples from the dams at weaning and the 16-week-old offspring were centrifuged and the resulting serum was collected for $\mathrm{Cr}$ analysis. Serum $\mathrm{Cr}$ concentrations were measured using an atomic absorption spectrometer (Atomic Absorption Spectrophotometer; Hitachi, Ltd., Tokyo, Japan).

Measurement of body weight and blood glucose levels. Body weight and blood glucose levels of dams were measured at weaning, and were measured in pups at birth, weaning and at 16 weeks of age. Tail blood glucose measurements were recorded using a blood glucose analyzer (Bayer Contour TS Glucometer; Bayer AG, Leverkusen, Germany).

Oral glucose tolerance test (OGTT). An OGTT was performed on dams at weaning and on pups at 16 weeks, after $12 \mathrm{~h}$ of food deprivation. The OGTT was performed in the morning. Glucose was introduced into the mice via oral gavage at a final dose of $2 \mathrm{~g} / \mathrm{kg}$ body weight. Blood samples were obtained from the tail vein 30, 60 and $120 \mathrm{~min}$ after glucose load in order to analyze glucose concentration using a glucometer (Bayer Contour TS Glucometer; Bayer AG). Glucose responses during the OGTT 


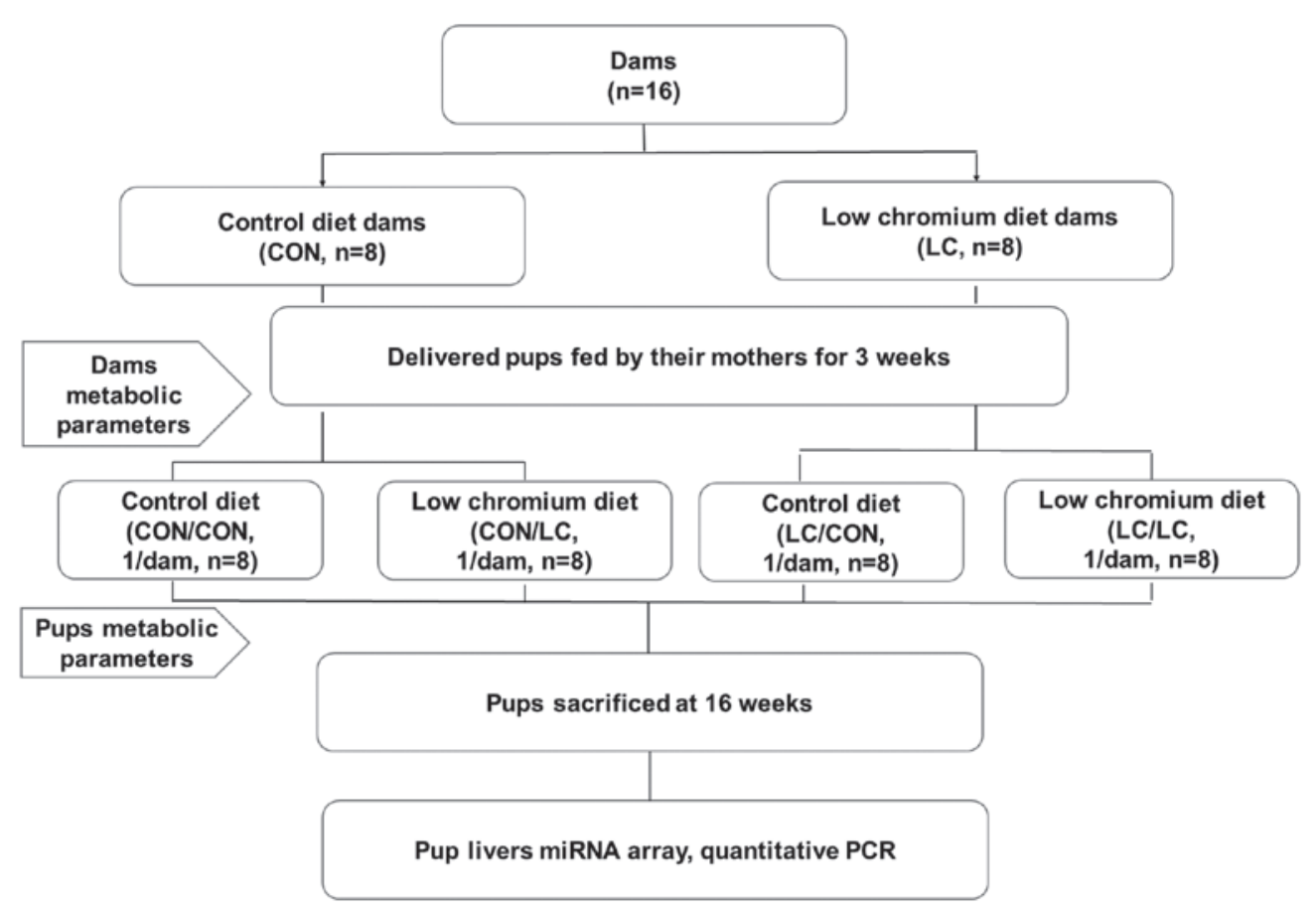

Figure 1. Animal experimental design. miRNA, microRNA; PCR, polymerase chain reaction.

were evaluated by estimating the total area under the curve (AUC) using the trapezoidal method.

Serum insulin levels and homeostasis model assessment of IR (HOMA-IR) index. After $12 \mathrm{~h}$ of food deprivation, tail blood samples were obtained and centrifuged. Serum insulin concentration was quantified using an ELISA method (mouse insulin kit; EZRMI-13K; EMD Millipore, Billerica, MA, USA) according to the manufacturer's protocol. Serum glucose was measured by an enzyme end-point method (Roche Diagnostics, GmnH, Mannheim, Germany). HOMA-IR was calculated as follows: Fasting serum glucose $\mathrm{x}$ fasting serum insulin/22.5.

RNA extraction, cDNA synthesis and miRNA array hybridization. Total liver RNA was isolated using the mirVana ${ }^{\mathrm{TM}}$ RNA Isolation kit (Ambion; Thermo Fisher Scientific, Inc., Waltham, MA, USA) according to the manufacturer's protocol. All RNA samples were examined for the absence of DNA and RNA degradation by denaturing agarose gel electrophoresis. Total RNA was then reverse transcribed using the PrimeScript Reverse Transcription (RT) kit (Takara Bio, Inc., Shiga, Japan) according to the manufacturer's protocol. The expression of miRNAs contained in miRBase version 20.0 (released in June, 2013; http://www.mirbase.org) was analyzed by microarray using the Affymetrix Multispecies miRNA 4.0 array (Affymetrix; Thermo Fisher Scientific, Inc.) according to the manufacturer's protocol. Raw data were normalized, processed, and analyzed for statistical significance using Student's t-test and post hoc Tukey's test was applied for a multiple testing correction.

miRNA quantitative polymerase chain reaction ( $q P C R$ ) validation. Total RNA obained using the mirVana ${ }^{\mathrm{TM}}$ RNA Isolation kit mentioned above was quantified using a NanoDrop 1000, and then reverse transcribed using TaqMan MicroRNA RT kit and RT primers from the respective TaqMan MicroRNA assay kit.
qPCR was performed on an ABI 7900 thermocycler using the TaqMan Universal PCR Master Mix and TaqMan probes from the TaqMan MicroRNA assay kit (all from Applied Biosystems; Thermo Fisher Scientific, Inc.). Taqman probes were synthesized according to miRNA mature sequneces (Table III). Amplification was performed at $95^{\circ} \mathrm{C}$ for $10 \mathrm{~min}$, followed by 40 cycles of $95^{\circ} \mathrm{C}$ for $15 \mathrm{sec}$ and $60^{\circ} \mathrm{C}$ for $60 \mathrm{sec}$. The reaction conditions were $16^{\circ} \mathrm{C}$ for $30 \mathrm{~min}, 42^{\circ} \mathrm{C}$ for $30 \mathrm{~min}$ and $85^{\circ} \mathrm{C}$ for $5 \mathrm{~min}$. Tissue miRNA levels were normalized to endogenous U6.

miRNA target gene bioinformatics analysis. miRTarBase database version 6.0 (released September, 2015; http://mirtarbase. mbc.nctu.edu.tw/) was used to perform bioinformatics analysis of the differentially expressed miRNAs and their validated target sequences (20).

miRNA target genes qPCR analysis. Total RNA extracted from the liver samples using the mirVana ${ }^{\mathrm{TM}}$ RNA Isolation kit mentioned above was reverse-transcibed by Superscript II (Invitrogen; Thermo Fisher Scientific, Inc.). qPCR analysis of gene expression was performed using an ABI Prism 7500 system (Applied Biosystems; Thermo Fisher Scientific, Inc.). The primers used were obtained from Applied Biosystems; Thermo Fisher Scientific, Inc. (Table II). Each PCR sample contained $20 \mathrm{ng}$ reverse-transcribed RNA, which was analyzed using SYBR-Green PCR Master Mix (Applied Biosystems; Thermo Fisher Scientific, Inc.). The following reaction conditions were used: $48^{\circ} \mathrm{C}$ for $30 \mathrm{~min}, 95^{\circ} \mathrm{C}$ for $15 \mathrm{~min}, 40$ cycles of $95^{\circ} \mathrm{C}$ for $15 \mathrm{sec}, 55^{\circ} \mathrm{C}$ for $1 \mathrm{~min}$. Target mRNA expression was normalized to $\beta$-actin expression and are expressed as a relative value using the cycle quantification $(\mathrm{Cq})$ method $\left(2^{-\Delta \Delta \mathrm{Cq}}\right)(21)$.

miRNA target genes pathway analysis. To fully clarify the biological functions of the target genes of differentially expressed miRNAs, Gene Ontology (GO) classifications and 
Table II. Quantitative polymerase chain reaction primers .

\begin{tabular}{llllc}
\hline $\begin{array}{l}\text { Gene } \\
\text { symbol }\end{array}$ & \multicolumn{1}{c}{ ID } & \multicolumn{1}{c}{ Forward } & \multicolumn{1}{c}{ Reverse } & $\begin{array}{c}\text { Product } \\
\text { length (bp) }\end{array}$ \\
\hline Akt1 & NM_009652 & 5'-CAGTTTGAGACCACACAT-3' & 5'-GCGTCAGTCCTTAATAGTT-3' & 75 \\
$P d p k$ l & NM_011062 & 5'-GGAATTGAATAGTGAGGTT-3' & 5'-AATGATGAATGTTGTATGTG-3' & 114 \\
$P i k 3 c a$ & NM_008839 & 5'-TGTGGCATCTGAGTATCT-3' & 5'-TGTGGCATCTGAGTATCT-3' & 132 \\
$P i k 3 r 1$ & NM_001077495 & 5'-ATTAAGGTTCTGTGGATTC-3' & 5'-GCTATGCTGTATCTATCTG-3' & 80 \\
$P i k 3 r 3$ & NM_181585 & 5'-ATTGGAATACCTGTGACT-3' & 5'-TGACTTACTTGGAAGAGAT-3' & 148 \\
Slc2a4 & NM_009204 & 5'-TATGTTGCGGATGCTATG-3' & 5'-TTAGGAAGGTGAAGATGAAG-3' & 82 \\
\hline
\end{tabular}

Akt1, thymoma viral proto-oncogene 1; Pdpk1,3-phosphoinositide dependent protein kinase 1; Pik3ca, phosphatidylinositol 3-kinase, catalytic, $\alpha$ polypeptide; Pik3rl, phosphatidylinositol 3-kinase, regulatory subunit, polypeptide (p85 $\alpha$ ); Pik3r3, phosphatidylinositol 3-kinase, regulatory subunit, polypeptide 3 (p55); Slc2a4, solute carrier family 2 member 4.

Kyoto Encyclopedia of Genes and Genomes (KEGG) pathways were determined, using Database for Annotation, Visualization and Integrated Discovery software (http://david.abcc.ncifcrf. gov/) (22). Interactions between miRNAs and mRNAs were analyzed by Cytoscape (http://www.cytoscape.org) (23).

Cell culture and treatments. HepG2 human liver cancer cells (American Type Culture Collection, Manassas, VA, USA) were maintained in Dulbecco's modified Eagle's medium supplemented with $10 \%$ fetal bovine serum, $1 \%$ pencillin-strepomycin, 2 mM glutamine (all from Lonza Group, Ltd., Basel, Switzerland) and $25 \mathrm{mM}$ HEPES (Sigma-Aldrich; Merck KGaA, Darmstadt, Germany) in a humidified incubator containing $5 \% \mathrm{CO}_{2}$ at $37^{\circ} \mathrm{C}$. HepG 2 cells $\left(2 \times 10^{5}\right.$ cells $\left./ \mathrm{ml}\right)$ were transfected with miR-327, miR-223-3p, miR-446f-3p (mature sequences in Table III; $10 \mu \mathrm{M}$; Ambion; Thermo Fisher Scientific, Inc.) or negative control oligoduplex (Ambion) using transfection reagent (Lipofectamine ${ }^{\circledR}$ RNAiMAX; Invitrogen; Thermo Fisher Scientific, Inc.) for $12 \mathrm{~h}$ at $37^{\circ} \mathrm{C}$. After $24 \mathrm{~h}$, cells were harvested for RNA preparation, and to measure the relative expression levels of miR-327, miR-223-3p, miR-446f-3p, thymoma viral proto-oncogene 1 (Akt1), 3-phosphoinositide dependent protein kinase 1 (Pdpkl), phosphatidylinositol 3-kinase, catalytic, $\alpha$ polypeptide ( $\mathrm{Pik} 3 \mathrm{ca}$ ), phosphatidylinositol 3-kinase, regulatory subunit, polypeptide (Pik3r1), phosphatidylinositol 3-kinase, regulatory subunit, polypeptide 3 (Pik3r3) and solute carrier family 2 member 4 [Slc2a4, also known as glucose transporter 4 (Glut4)].

Data statistical analysis. All numerical results are expressed as the means \pm standard deviation of the indicated number of experiments. Student's t-test was used to compare unpaired samples, and One-way analysis of variance followed by post hoc Tukey test was used for multiple comparisons. GraphPad Prism software version 5.0 (GraphPad Software, Inc., La Jolla, CA, USA) was used to analyze the data. $\mathrm{P}<0.05$ was considered to indicate a statistically significant difference.

\section{Results}

Dams. Serum Cr concentrations were lower in the LC group compared with in the CON group $(0.36 \pm 0.03$ vs. $0.74 \pm 0.14 \mathrm{ng} / \mathrm{ml}$, $\mathrm{P}<0.01)$. Body weight, fasting blood glucose, blood glucose levels, AUC of glucose concentration during OGTT, serum insulin levels and HOMA-IR index in LC dams were similar to CON dams at the end of lactation (data not shown).

\section{Offspring}

Effect of maternal LC diet on serum $C r$ in offspring. At 16 weeks of age, serum $\mathrm{Cr}$ in the $\mathrm{LC} / \mathrm{LC}(0.31 \pm 0.02 \mathrm{ng} / \mathrm{ml})$ and CON/LC groups $(0.27 \pm 0.01 \mathrm{ng} / \mathrm{ml})$ was significantly reduced compared with in the CON/CON $(0.69 \pm 0.03 \mathrm{ng} / \mathrm{ml})$ and LC/CON groups $(0.72 \pm 0.09 \mathrm{ng} / \mathrm{ml}, \mathrm{P}<0.01)$.

Effects of maternal LC diet on body weight of offspring. At birth, 3 and 16 weeks of age, body weight of male pups was not markedly different among the various groups.

Effects of maternal LC diet on fasting blood glucose in offspring. At week 3, no significant differences in fasting blood glucose were noted among all groups. At week 16, fasting blood glucose in the CON/LC $(8.32 \pm 0.72 \mathrm{mmol} / \mathrm{l}), \mathrm{LC} / \mathrm{CON}$ $(8.19 \pm 0.92 \mathrm{mmol} / \mathrm{l})$ and LC/LC groups $(8.52 \pm 0.73 \mathrm{mmol} / \mathrm{l})$ was significantly higher compared with in the CON/CON group (5.83 $\pm 0.07 \mathrm{mmol} / \mathrm{l}, \mathrm{P}<0.05)$.

Effects of maternal LC diet on glucose tolerance in offspring. To determine whether maternal LC diet disturbs glucose tolerance in offspring, an OGTT was conducted at 16 weeks of age. After a bolus of oral glucose, blood glucose levels were significantly increased in the offspring of the $\mathrm{CON} / \mathrm{LC}, \mathrm{LC} / \mathrm{LC}$ and $\mathrm{LC} / \mathrm{CON}$ groups compared with in the CON/CON group at 30,60 and $120 \mathrm{~min}(\mathrm{P}<0.05$ or $\mathrm{P}<0.01)$. AUC of blood glucose concentration was increased in the $\mathrm{CON} / \mathrm{LC}(51.43 \pm 4.38 \mathrm{mmol} / \mathrm{l} / \mathrm{h}), \mathrm{LC} / \mathrm{LC}(53.18 \pm 4.83 \mathrm{mmol} / \mathrm{l} / \mathrm{h})$ and $\mathrm{LC} / \mathrm{CON}$ groups $(48.85 \pm 5.28 \mathrm{mmol} / \mathrm{l} / \mathrm{h})$ compared with in the $\mathrm{CON} / \mathrm{CON}$ group $(26.23 \pm 3.14 \mathrm{mmol} / \mathrm{l} / \mathrm{h}, \mathrm{P}<0.01)$.

Effects of maternal LC diet on serum insulin and HOMA-IR index in offspring. Serum fasting insulin levels were significantly increased in the CON/LC $(11.8 \pm 1.84 \mu \mathrm{IU} / \mathrm{ml})$ and LC/LC groups $(13.2 \pm 1.8 \mu \mathrm{IU} / \mathrm{ml})$ compared with in the $\mathrm{CON} / \mathrm{CON}$ group $(6.4 \pm 0.53 \mu \mathrm{IU} / \mathrm{ml}, \mathrm{P}<0.05)$. HOMA-IR index in the LC/CON (3.8 \pm 0.37$), \mathrm{CON} / \mathrm{LC}(3.1 \pm 0.24)$ and $\mathrm{LC} / \mathrm{LC}$ groups $(3.8 \pm 0.26)$ was higher than that in the $\mathrm{CON} / \mathrm{CON}$ group $(2.1 \pm 0.13, \mathrm{P}<0.01)$ (data not shown).

miRNA array. Affymetrix miRNA 4.0 array contains all known mouse and rat miRNA annotated in the miRBase 20.0 sequence 


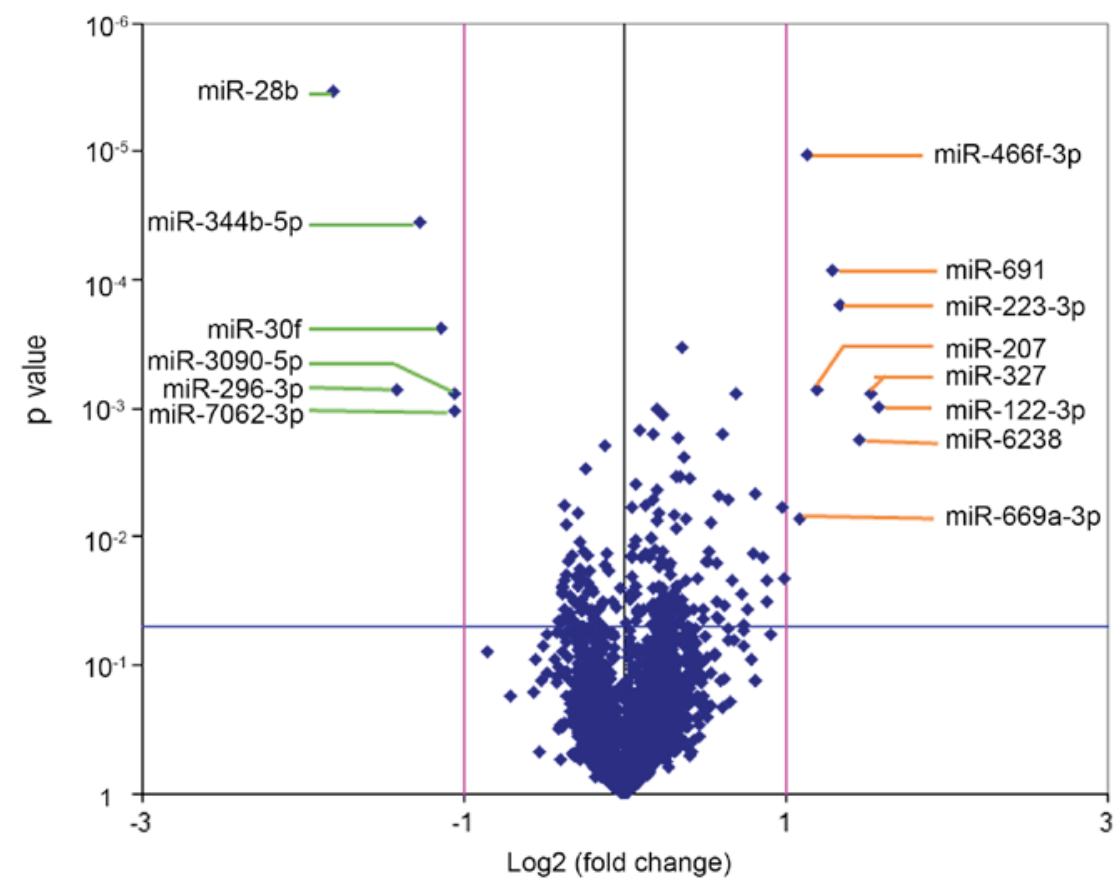

Figure 2. Volcano plot of the miRNA microarray analysis. Volcano plots are used to visualize differential expression patterns between two groups. Vertical lines correspond to 2 -fold up- and downregulation, and the horizontal line represents $\mathrm{P}=0.05$. $\mathrm{miR} / \mathrm{miRNA}$, microRNA.

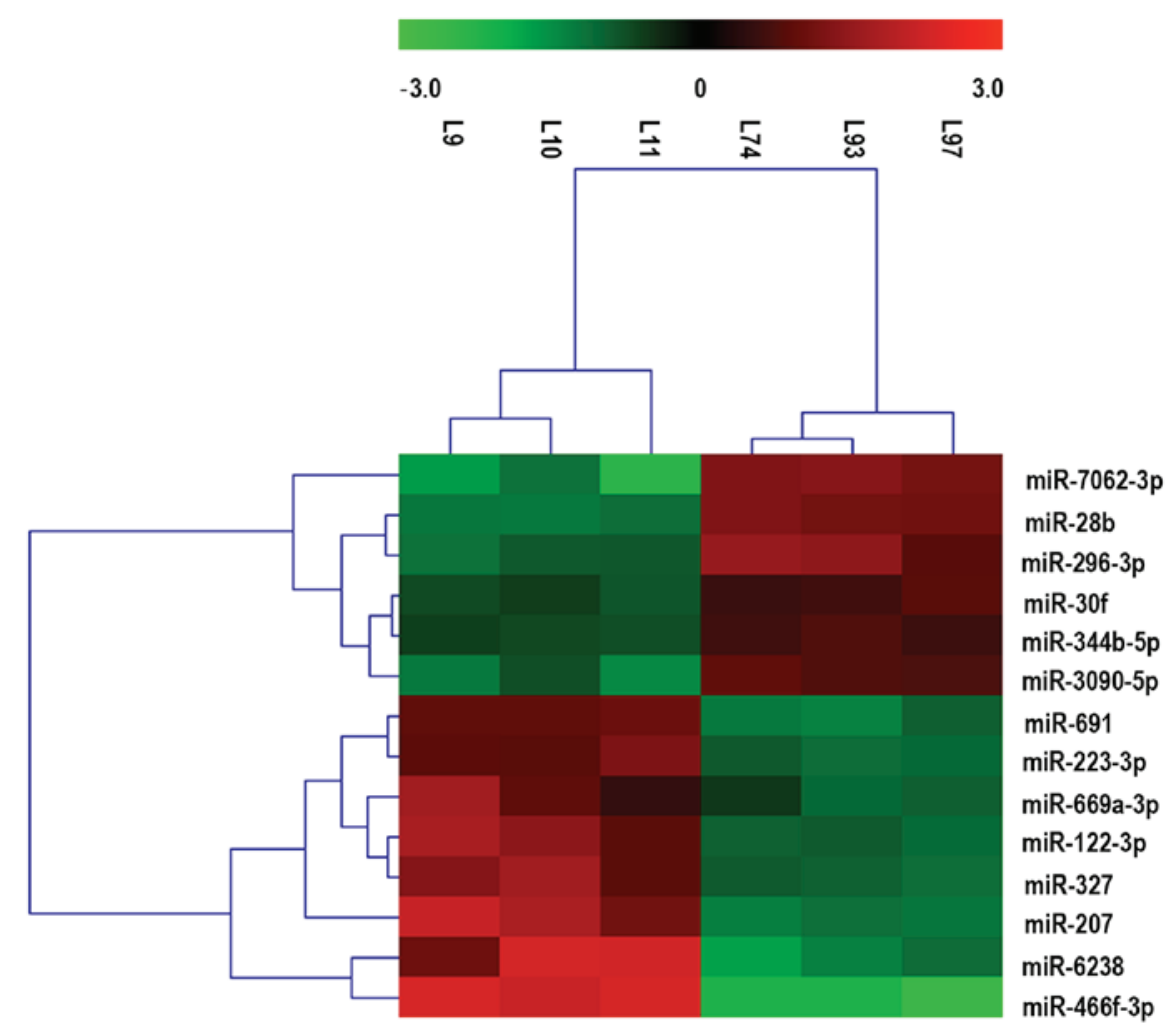

Figure 3. Hierarchical clustering of the differentially expressed miRNAs in the LC/CON group compared with in the CON/CON group. Left, miRNA clustering tree; top, sample clustering tree. Red indicates high relative expression, and green indicates low relative expression. L9, L10 and L11 belong to the LC/CON group; L74, L93 and L97 belong to the CON/CON group. CON, control; LC, low chromium; miRA/miR, microRNA.

database. A volcano plot was generated to identify differential expression between the LC/CON and CON/CON groups, as shown in Fig. 2. The cluster and heat maps of all detected differentially expressed miRNAs were plotted to better demonstrate differential miRNA expression between the two groups (Fig. 3).
The miRNA array yielded 14 significantly differentially expressed miRNAs (Table III). Among the 14 significantly differentially expressed miRNAs, 8 miRNAs were upregulated (mmu-miR-122-3p, mmu-miR-6238, mmu-miR-669a-3p, mmu-miR-691, mmu-miR-223-3p, mmu-miR-327, 
Table III. Significantly differentially expressed miRNAs in the livers of offspring from dams fed a low chromium diet.

\begin{tabular}{|c|c|c|c|c|}
\hline MicroRNA & Fold change & Regulation & P-value & Mature sequences \\
\hline mmu-miR-30f & 0.4549 & Down & 0.000241 & GUAAACAUCCGACUGAAAGCUC \\
\hline mmu-miR-344b-5p & 0.4134 & Down & $3.56 \times 10^{-5}$ & AGUCAGGCUCCUGGCUAAAGUUC \\
\hline mmu-miR-28b & 0.2847 & Down & $3.45 \times 10^{-6}$ & AGGAGCUCACAAUCUAUUUAG \\
\hline mmu-miR-296-3p & 0.3765 & Down & 0.000718 & GAGGGUUGGGUGGAGGCUCUCC \\
\hline mmu-miR-7062-3p & 0.4833 & Down & 0.001047 & ACUAACUUCUCCUGGCCCCACAG \\
\hline mmu-miR-3090-5p & 0.4811 & Down & 0.000773 & GUCUGGGUGGGGCCUGAGAUC \\
\hline mmu-miR-122-3p & 3.0022 & Up & 0.000983 & AAACGCCAUUAUCACACUAA \\
\hline mmu-miR-6238 & 2.7639 & Up & 0.001775 & UUAUUAGUCAGUGGAGGAAAUG \\
\hline mmu-miR-669a-3p & 2.1272 & Up & 0.007318 & ACAUAACAUACACACACACGUAU \\
\hline mmu-miR-691 & 2.4515 & Up & $8.43 \times 10^{-5}$ & AUUCCUGAAGAGAGGCAGAAAA \\
\hline mmu-miR-223-3p & 2.5277 & Up & 0.000156 & UGUCAGUUUGUCAAAUACCCCA \\
\hline mmu-miR-327 & 2.9025 & Up & 0.000762 & ACUUGAGGGGCAUGAGGAU \\
\hline mmu-miR-207 & 2.2873 & Up & 0.000728 & GCUUCUCCUGGCUCUCCUCCCUC \\
\hline mmu-miR-466f-3p & 2.1942 & Up & $1.06 \times 10^{-5}$ & CAUACACACACACAUACACAC \\
\hline
\end{tabular}

miR/miRNA, microRNA.
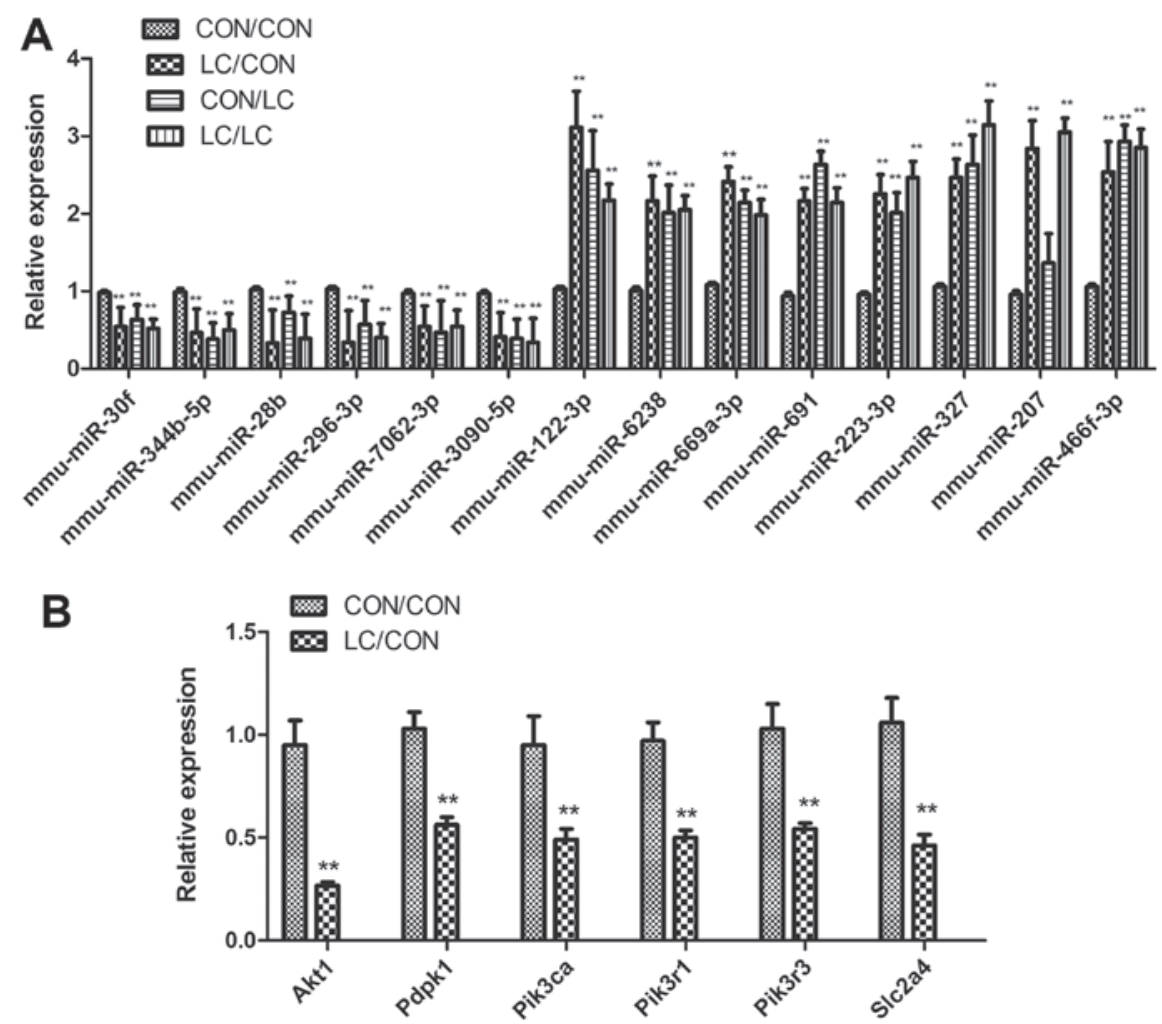

Figure 4. Differential miRNA expression underwent (A) qPCR validation and (B) target genes of the differentially expressed miRNAs were detected by qPCR. Data are presented as the means \pm standard deviation, $\mathrm{n}=8$. ${ }^{* *} \mathrm{P}<0.01$ vs. the CON/CON group. Aktl, thymoma viral proto-oncogene 1; CON, control; LC, low chromium; miR/miRNA, microRNA; Pdpkl, 3-phosphoinositide dependent protein kinase 1; Pik3ca, phosphatidylinositol 3-kinase, catalytic, $\alpha$ polypeptide; Pik3rl, phosphatidylinositol 3-kinase, regulatory subunit, polypeptide (p85 $\alpha$ ); Pik3r3, phosphatidylinositol 3-kinase, regulatory subunit, polypeptide 3 (p55); qPCR, quantitative polymerase chain reaction; Slc2a4, solute carrier family 2 member 4.

mmu-miR-207 and mmu-miR-466f-3p), and 6 miRNAs were downregulated (mmu-miR-30f, mmu-miR-344b-5p, mmu-miR-28b, mmu-miR-296-3p, mmu-miR-7062-3p and mmu-miR-3090-5p). Differentially expressed miRNAs were defined by a fold change $\geq 2$, up- or downregulated and $\mathrm{P}<0.05$. $q P C R$. qPCR was used to confirm differential miRNA expression and to detect the target genes of differentially expressed miRNAs (Fig. 4). The differential expression of miRNAs was confirmed by RT-qPCR; the results were consistent with the array analysis (Fig. 4A). 
Table IV. Validated target genes of 8 differentially expressed miRNAs ${ }^{\mathrm{a}}$.

\begin{tabular}{|c|c|c|}
\hline miRNA & Regulation & Target genes \\
\hline mmu-miR-28b & Down & $\begin{array}{l}\text { Slc7a2, Nr3c1, Timm17b, Timm17b, 2010109I03Rik, Tars2, Slx1b, Tns4, Prss43, } \\
\text { Pld5, Ccdc78, Tmppe }\end{array}$ \\
\hline mmu-miR-296-3p & Down & $\begin{array}{l}\text { Ctla2a, Gna14, Igf1r, Dusp8, Tfam, Tnf, Cxcl11, Car5b, Nsa2, Clvs1, Phf14, Phf14, } \\
\text { Braf, Mcmdc2, Prss43 }\end{array}$ \\
\hline mmu-miR-669a-3p & Up & $\begin{array}{l}\text { Cry2, Cd47, Kras, Shh, Zfp37, Rnf11, Ccdc82, 2610002M06Rik, Wdfy3, 1700019G17Rik, } \\
\text { Fam159b, Cml2, Arrdc3, Sipa111, Csde1, Gm4944, Gm7616 }\end{array}$ \\
\hline mmu-miR-691 & Up & $\begin{array}{l}\text { Cbln2, Cdk7, Inpp5d, Ndrg1, Sp4, Pcgf2, Scd3, Arl6ip1, Wtap, Atp2b1, 1700025G04Rik, } \\
\text { Sik3, Dapl1, Adamts11, Utp23, Cnnm1, Hlcs }\end{array}$ \\
\hline mmu-miR-223-3p & Up & $\begin{array}{l}\text { Abca1, Slc33a1, Macf1, Ank3, Prdx6, Aplp2, Atp9a, Atrn, Pcdh15, Casp9, Cbx5, Cckbr, } \\
\text { Cdkn1b, Celf1, Dag1, Ddn, Enc1, Eps15, Ercc3, F2r, F3, Fmn1, Srgap2, Fut9, } \\
\text { Gabra6, Gabrg1, Gria2, Grin2b, Trip12, Gzmb, Sdc2, Igf1r, Itgb1, F11r, Kcnj10, Kcnj3, } \\
\text { Kif1b, Klf12, Kpna1, Kras, Ktn1, Ldlr, Zbtb7a, Smad5, Mecp2, Mef2c, Mt2, Map1b, Myo1c, } \\
\text { Napb, Nf1, Nfib, Ntrk2, Dusp8, Pdgfra, Enpp1, Pdpk1, Pea15a, Peg3, Pgr, Phka1, Pik3r3, } \\
\text { Prkcb, Pkia, Plod1, Plxna2, Prkar2b, Prnp, Ptpru, Pvt1, Rest, Rgs2, Xpr1, Rtn3, Scd1, Scn8a, } \\
\text { Sdpr, Sept8, Serf1, Sox6, Stat3, Syn1, Syp, Syt4, Tnfaip1, Utrn, Vim, Xpc, Ywhaz, } \\
\text { Zfp26, Akt3, Apc2, Ets1, Gm16515, Gnpda1, Mapk10, B3galt1, B3galnt1, Syn3, Trp53bp1, } \\
\text { Amot, Nrep, Ubfd1, Mga, Rnf11, Fbxw7, Tmod2, Mfhas1, Tmx4, Sgsm1, Pgrmc1, Stk39, } \\
\text { Vti1a, Gria3, Rragc, Ptbp2, Ak3, Ptpn9, Dbn1, Socs5, Foxo3, Rnf14, Mbnl1, Jph3, Ext12, } \\
\text { Fam13a, Usp14, Gprc5b, Txndc12, Tmem57, Cyb5b, Rnf220, Asf1b, Ddx17, Slc25a46, } \\
\text { Polr3g, Gpatch2, Tmem33, Camsap2, Atp2b1, Tmem178, Ndnf, Ankra2, Pdrg1, } \\
\text { 1190002N15Rik, Abhd13, Zfp467, Sec62, Ttc9, Zfp619, Afap1, Gcc2, Draxin, Spag9, } \\
\text { Fmn12, Ap2b1, Klh15, March8, Pdia6, Antxr2, Zcchc24, Dusp11, Slc37a3, Fbx120, Usp36, } \\
\text { Ttc4, Mvb12b, Dnajc6, Tppp, Ccnt2, 3110043O21Rik, Gpbp1, 1700012B15Rik, Lonrf3, } \\
\text { Arl5a, Rap2a, Car12, Paqr4, Clasp2, Usp42, Golga1, Atg1611, Kidins220, Myh10, Yipf6, } \\
\text { Trappc6b, Mvp, Acap2, Ankrd17, Cacng7, Smarcd2, Pard6g, Vang12, Trim9, Gopc, } \\
\text { Dgcr8, Cadm3, Pds5b, Tbc1d14, Akap9, Inpp5f, Bbs4, Dnm3, Arrdc3, Epdr1, Ypel1, } \\
\text { Tm9sf3, Grm7, Foxp1, Dnajc9, Jmjd1c, Fam73b, Ank2, Zfp91, Shroom2, Hlcs, Derl2, } \\
\text { Fam57a, Ube3b, Hpcal4, Mtmr4, Zfp704, Glcci1, Mlc1, Cd9912, Prpf8, Phf21a, } \\
\text { Mical3, C2cd2, Rsrc2, Cblb, Daam1, Cpeb3, Prrt3, Kbtbd2, Dennd6a, Kmt2a, Gnptg, } \\
\text { L3mbt12, Rhbd11, Vezt, Tom112, Hlf, 4933426M11Rik, Pacs2, Rasa1, Srek1, Spata13, Xkr6, } \\
\text { Pcdh17, Golgb1, Cep120, Fam78b, Cdc42bpa, Ino80d, Camk1d, Sestd1, Zmynd8, } \\
\text { 4932438A13Rik, Tnrc18, Vopp1, Eva1a, Mob1a, Ppp4r2, Luzp2, Arglu1, Psd3, Atg4d, } \\
\text { Ago1, Nxt2, Em16, Usp32, Pnma2, Phf2011, Mb21d2, Gpr158, Rbm45, Lrrc55, Tspyl3, Podn, } \\
\text { Wasf2, Stard13, Kat6a, Mllt6, Lgi2, Lsamp, Robo2, Caskin1, Luzp1, Nceh1, Zbtb39, Mdga2, } \\
\text { Zhx3, Pitpnm3, Fnip2, Tmem67, Slc5a6, Dock1, Ksr2, Pcdhac1, Nefh, Lonrf2, Rbm33, }\end{array}$ \\
\hline
\end{tabular}

mmu-miR-327 Up

mmu-miR-207 Up

mmu-miR-466f-3p Up
Znrf3, Adcy1, Bdp1, Tnik

Clk4, Mef2c, Ptprb, Slc2a4, Cntnap2, Fam168b, Cep68, Evi2b, Zmym5, Rasal2, Rab39, Pld5 Adcy6, Cplx2, Emp2, Gda, Itgam, Lpl, Nab1, Nrl, Pigr, Prlr, Slc22a12, Ubtf, Txn14a, Preb, Sh3bgrl, Rab3c, Asc14, Prr51, Steap2, Crebrf, BC021785, Plcl1, Ntm, Gm4944, Prkag3,

Dzank1, Oacyl

Adam9, Adcyap1r1, Akap2, Ap1g1, Xiap, Arf3, Zfhx3, Atf2, Atp7a, Pcdh15, Chic1, Bsn, Chek1, Cnr1, Cpd, Cry2, Drp2, E2f1, Efnb2, Eif2s1, Dmtn, Epha4, Ereg, Eya1, Fech, Gnb4, Grb2, Grik3, Hist1h1d, Foxq1, Hiat1, Hk2, Id2, Ids, Il17ra, Inpp5d, Jag2, Kcna4, Klra2, Hivep3, Lox13, Mef2a, Mmp12, Mmp11, Mtf1, Nck2, Nfatc2, Nodal, Ints6, Npy 1r, Slc11a2, Nrf1, Nrg3, Nrp2, Pappa, Piga, Pik3ca, Pik3r1, Prkcb, Plau, Prrx1, Ppargc1a, Pter, Ptp4a2, Ptprk, Rai1, Rgl1, Msr1, Cxcl5, Sin3a, Slc12a2, Smarca4, Spta1, Tcf4, Tcf712, Tcte1, Tll1, Tlr4, Tnfrsf11a, Trhr, Unc5c, Uty, Vegfa, Wfs1, Xk, Pcgf2, Zbtb7b, Ikzf2, Gtf2h2, Dnajb6, Rnasel, Sept3, Taf7, Ldb3, Cops7b, Azi2, Syt14, Csnk1e, Prl5a1, Vps26a, Fbx117, Sept11, Hif3a, B3gnt2, Diap2, Usp27x, Pitpnb, Pias1, Gsk3b, Akap10, Plag1, Gmeb1, 
Table IV. Continued.

\begin{tabular}{|c|c|c|}
\hline miRNA & Regulation & Target genes \\
\hline & & $\begin{array}{l}\text { Tbx20, Piwil2, Usp29, Cd200r1, Pard6b, Fign, Ms4a4b, Wtap, Ms4a4c, Gpr85, Mrps24, } \\
\text { Dimt1, Haus2, Ccdc82, Atg10, Slc16a9, Slc25a46, Klhl13, Zfp169, Nacc2, Pdf, Golt1a, } \\
\text { Rraga, Fndc1, Anln, Synpo21, Phtf2, Casz1, Sash1, Mllt3, Tbc1d13, Rufy2, Prss22, Pgm211, } \\
\text { Foxn3, Tmem25, Smug1, Mtfr2, Nup93, Cyb5r1, Ccpg1, Slc16a10, Ints8, Zfp444, Tmem161b, } \\
\text { Zfp839, Appl1, Insig2, Larp1, Ddit41, Rap2b, Ehhadh, Ubap21, Ifitm7, Mdga1, Uhrf1bp11, } \\
\text { Dusp18, Arhgap12, Fam227a, Dcaf17, Slx1b, Ppp1r14c, Abca6, Rtf1, Fam101b, B3gat1, } \\
\text { Bcas1, Crebrf, Csrnp3, Cdk19, Clca2, Tbl1xr1, Tfcp211, Tdrd1, Pde4dip, Tas1r3, Mcam, } \\
\text { Spock2, Arid4b, Hecw1, Ubac1, Lrrc19, Zfand2a, Zfp518b, Fbx114, Adamts9, Dennd4a, } \\
\text { Slc6a8, Dnm3, Unc5a, Ssr1, Mylk, Ston2, Trmt10a, Fam212b, Prr5, Lmx1a, Scn2a1, Erc1, } \\
\text { Slc28a3, Rasa2, Pofut1, Asb13, Bbc3, Edaradd, Rwdd4a, Ash11, Wfdc12, Ell2, Bzrap1, } \\
\text { Prrg3, Ido2, Mkx, Alkbh1, Fam46a, Gabpb2, Armc2, Arhgef11, Tnrc6b, Tet2, Fam168b, } \\
\text { BC003965, Tmprss13, Phactr2, Kctd11, Spag7, Evi2b, Rnf157, Nploc4, Stxbp6, Cep170b, } \\
\text { Gxylt1, Tbc1d24, Cep76, Plc11, Rabgap1, Cytip, Atp5g3, Mavs, Hnrnpa3, Bcl2115, Slc6a17, } \\
\text { Strip1, Cdc14a, Tmem201, Lin54, Oas1e, BC030336, Fus, Tpte, Psd3, Ankle1, Fhod1, Stard8, } \\
\text { Ofd1, Zc3h12d, C2cd4c, Ric8b, Btn19, Pfas, Fam160b2, Apol8, Rtp1, 9930021J03Rik, } \\
\text { Zbtb34, Zbtb6, Lzts3, Dzank1, Zfp462, Klh121, Rsbn11, Ugt2b35, Ppm1k, Lonrf1, Cwf1912, } \\
\text { Zscan4c, Tbx22, Klf8, Atxn7, Oas3, Srgap3, Alkbh5, Greb1, Fam107a, Ctif, Nbeal1, Ss1811, } \\
\text { Lhfp14, Fat3, Bpifc, Trim12c, Fndc3a, Hif1an, Itpripl2, A630001G21Rik, Zbtb39, Adrbk2, } \\
\text { Hook3, Rnf152, Hist4h4, Ubn2, Fam169a, Sycp2, Ccdc169, Ar14c, Xpnpep3, Slfn5, Gfod1, } \\
\text { Mast4, Skint7, Zfp516, Acot11, Taok3, Opcml, Gpd11, Jhdm1d, Lin28b, Gm1587, Tbc1d22b, } \\
\text { Arl14epl, Gse1, Mtx3, Zfp874b, Gm13476, Ociad2, Tmem178b, Fam169b, Scrt2, Zscan4d, } \\
\text { Mast3, Zfp872, Zfp963, Zfp827, I830012O16Rik, AK010878 }\end{array}$ \\
\hline
\end{tabular}

${ }^{a}$ Validated target genes in miRTarBase database version 6.0. miR/miRNA, microRNA.

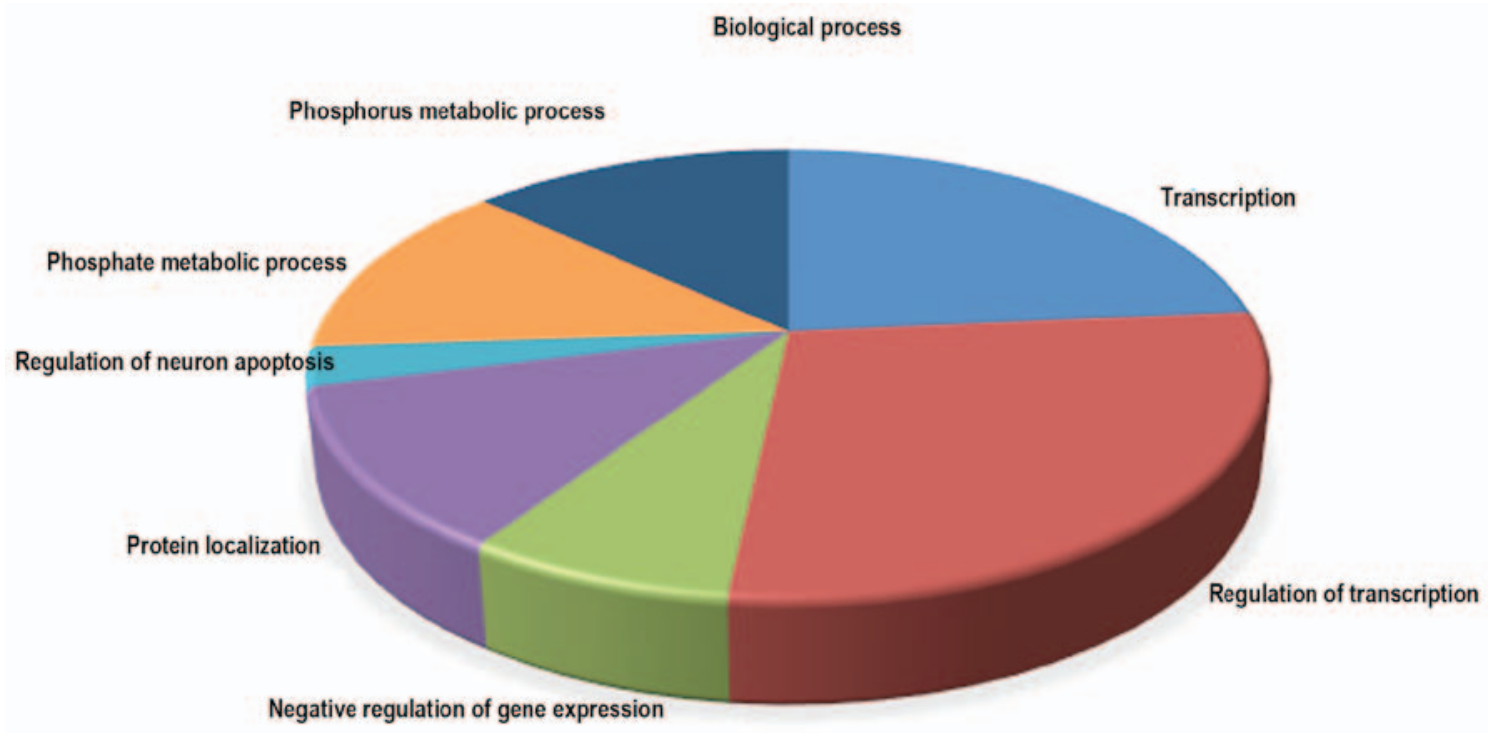

Figure 5. Gene Ontology analysis of the miRNA target genes in biological process.

Bioinformatics analysis. Based on the data from miRTarBase databases, 8 of the 14 dysregulated miRNAs have 732 validated target genes (Table IV). The significantly over-represented biological process, cellular component and molecular function GO terms $(\mathrm{P}<0.001)$ in the differentially expressed miRNA targets are presented in Table V. In biological process, the GO terms were involved with transcription, regulation of transcription, negative regulation of gene expression, protein localization, regulation of neuron apoptosis, phosphate metabolic process and phosphorus metabolic process (Fig. 5). A pathway analysis was conducted using the 8 differentially expressed miRNA targets; pathway title and the $\mathrm{P}$-value of the significant pathways $(\mathrm{P}<0.0001)$, are presented in Table VI. KEGG pathway analysis showed 
Table V. Gene Ontology terms significantly over-represented in the differentially expressed miRNA target genes in three separate domains $(\mathrm{P}<0.001)$.

\begin{tabular}{|c|c|c|c|c|}
\hline Term & DE Count & Fold enrichment & P-value & Ontology \\
\hline Transcription & 92 & 1.4456 & $2.06 \times 10^{-4}$ & Biological Process \\
\hline Regulation of transcription & 110 & 1.3753 & $2.80 \times 10^{-4}$ & Biological Process \\
\hline Negative regulation of gene expression & 30 & 2.0373 & $3.87 \times 10^{-4}$ & Biological Process \\
\hline Protein localization & 46 & 1.7009 & $5.17 \times 10^{-4}$ & Biological Process \\
\hline Regulation of neuron apoptosis & 11 & 3.8285 & $5.53 \times 10^{-4}$ & Biological Process \\
\hline Phosphate metabolic process & 51 & 1.6397 & $5.66 \times 10^{-4}$ & Biological Process \\
\hline Phosphorus metabolic process & 51 & 1.6397 & $5.66 \times 10^{-4}$ & Biological Process \\
\hline Transcription & 92 & 1.4456 & $2.06 \times 10^{-4}$ & Biological Process \\
\hline Plasma membrane & 140 & 1.3912 & $9.85 \times 10^{-6}$ & Cellular Component \\
\hline Cell fraction & 41 & 1.9865 & $4.33 \times 10^{-5}$ & Cellular Component \\
\hline Plasma membrane part & 86 & 1.5208 & $5.55 \times 10^{-5}$ & Cellular Component \\
\hline Insoluble fraction & 37 & 2.0236 & $7.68 \times 10^{-5}$ & Cellular Component \\
\hline Membrane fraction & 36 & 2.0384 & $8.46 \times 10^{-5}$ & Cellular Component \\
\hline Synapse & 24 & 2.1726 & $7.18 \times 10^{-4}$ & Cellular Component \\
\hline Neuron projection & 20 & 2.3573 & $8.54 \times 10^{-4}$ & Cellular Component \\
\hline Cell junction & 31 & 1.9046 & $9.00 \times 10^{-4}$ & Cellular Component \\
\hline Clathrin-coated vesicle & 12 & 3.3320 & $9.14 \times 10^{-4}$ & Cellular Component \\
\hline GTPase regulator activity & 30 & 2.3798 & $2.57 \times 10^{-5}$ & Molecular Function \\
\hline Nucleoside-triphosphatase regulator activity & 30 & 2.3409 & $3.49 \times 10^{-5}$ & Molecular Function \\
\hline Transition metal ion binding & 126 & 1.3835 & $5.71 \times 10^{-5}$ & Molecular Function \\
\hline Metal ion binding & 173 & 1.2868 & $6.86 \times 10^{-5}$ & Molecular Function \\
\hline Ion binding & 176 & 1.2812 & $7.24 \times 10^{-5}$ & Molecular Function \\
\hline Cation binding & 173 & 1.2752 & $1.15 \times 10^{-4}$ & Molecular Function \\
\hline GTPase activator activity & 19 & 2.8339 & $1.33 \times 10^{-4}$ & Molecular Function \\
\hline Protein tyrosine phosphatase activity & 13 & 3.6860 & $1.99 \times 10^{-4}$ & Molecular Function \\
\hline Zinc ion binding & 103 & 1.4012 & $2.31 \times 10^{-4}$ & Molecular Function \\
\hline Cytoskeletal protein binding & 30 & 2.0752 & $2.87 \times 10^{-4}$ & Molecular Function \\
\hline DNA binding & 89 & 1.4310 & $3.63 \times 10^{-4}$ & Molecular Function \\
\hline
\end{tabular}

DE count, differential expression count.

Table VI. Significant pathways of the dysregulated miRNA target genes $(\mathrm{P}<0.0001)$.

\begin{tabular}{|c|c|c|c|}
\hline Pathway & Count & $\begin{array}{c}\text { Fold } \\
\text { enrichment }\end{array}$ & P-value \\
\hline Endometrial cancer & 13 & 7.5104 & $7.94 \times 10^{-8}$ \\
\hline Colorectal cancer & 15 & 5.2398 & $6.60 \times 10^{-7}$ \\
\hline ErbB signaling pathway & 15 & 5.1796 & $7.64 \times 10^{-7}$ \\
\hline Non-small cell lung cancer & 12 & 6.6759 & $1.08 \times 10^{-6}$ \\
\hline Prostate cancer & 15 & 5.0069 & $1.17 \times 10^{-6}$ \\
\hline Insulin signaling pathway & 18 & 3.9185 & $2.41 \times 10^{-6}$ \\
\hline Glioma & 11 & 5.1634 & $3.99 \times 10^{-5}$ \\
\hline
\end{tabular}

that the target genes of differential expression miRNAs in LC/CON mice were mainly focused on insulin signaling pathway (Fig. 6). miRNAs and their target interactions are presented in Fig. 7.
miRNA target genes qPCR. Aktl, Pdpk1, Pik3ca, Pik3rl, Pik3r3 and Slc2a4 (Glut4) were downregulated in the LC/CON group compared with in the CON/CON group, as demonstrated by RT-qPCR (Fig. 4B).

miR-327, miR-223-3p and miR-466f-3p target the insulin signaling pathway in HepG2 cells. To determine whether miR-327, miR-223-3p and miR-466f-3p negatively regulate the insulin signaling pathway in vitro, HepG2 cells were transfected with miR-327, miR-223-3p and miR-466f-3p mimics. Slc2a4 expression was significantly downregulated in miR-327-transfected HepG2 cells ( $\mathrm{P}<0.01$; Fig. 8A). In miR-466f-3p-transfected HepG2 cells, Pik3ca and Pik3rl were significantly downregulated $(\mathrm{P}<0.01$; Fig. 8B). In miR-223-3p-transfected HepG2 cells, Akt1, Pdpk1 and Pik3r3 were significantly downregulated ( $\mathrm{P}<0.01$; Fig. $8 \mathrm{C})$.

\section{Discussion}

In the present study, a 13-week LC diet led to impaired fasting blood glucose and glucose tolerance, and IR in male mice. 
INSULIN SIGNALING PATHWAY

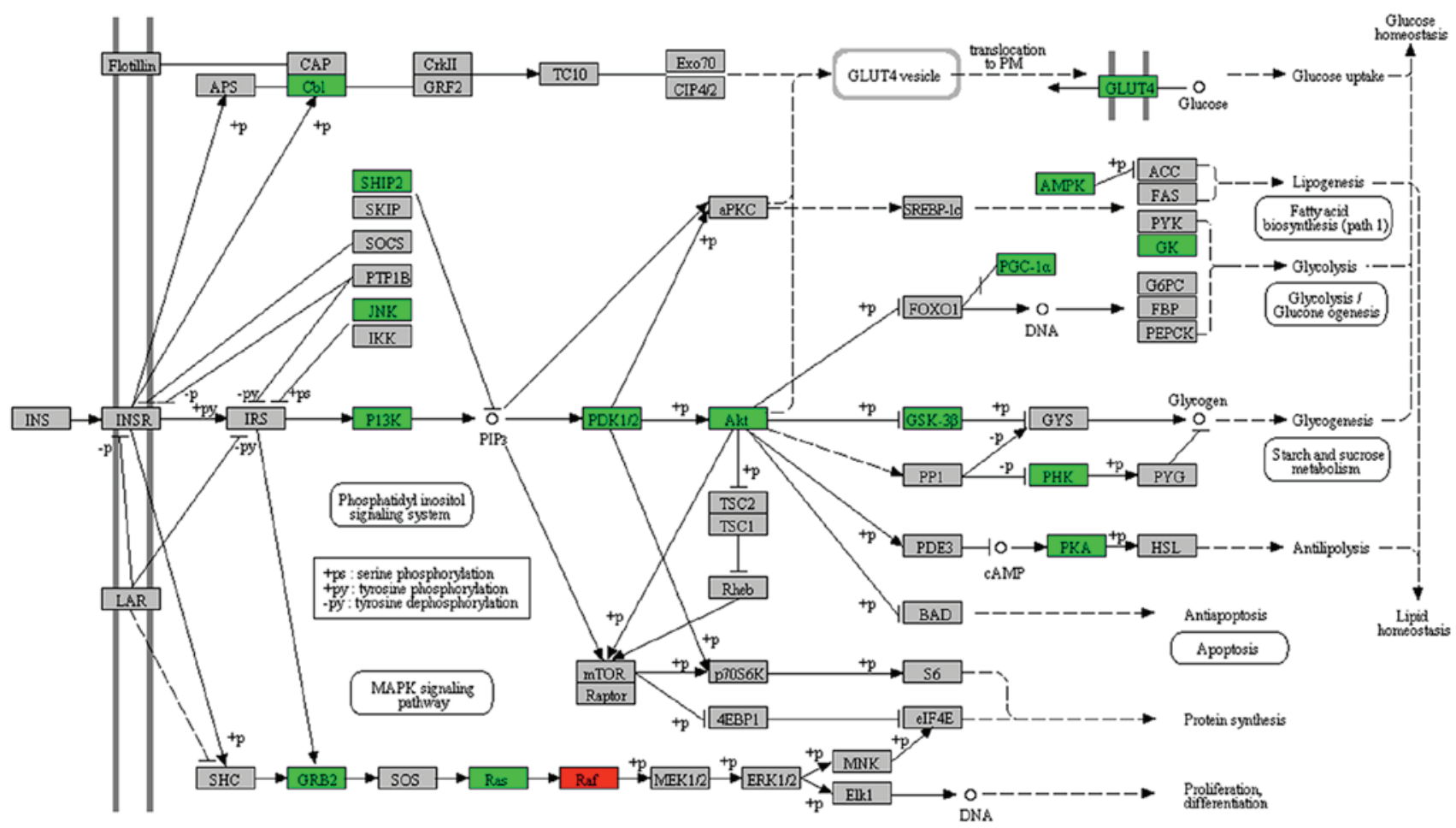

049108141415

Figure 6. Differentially expressed miRNA gene targets in the insulin signaling pathway. Red represents upregulation; green represents downregulation.
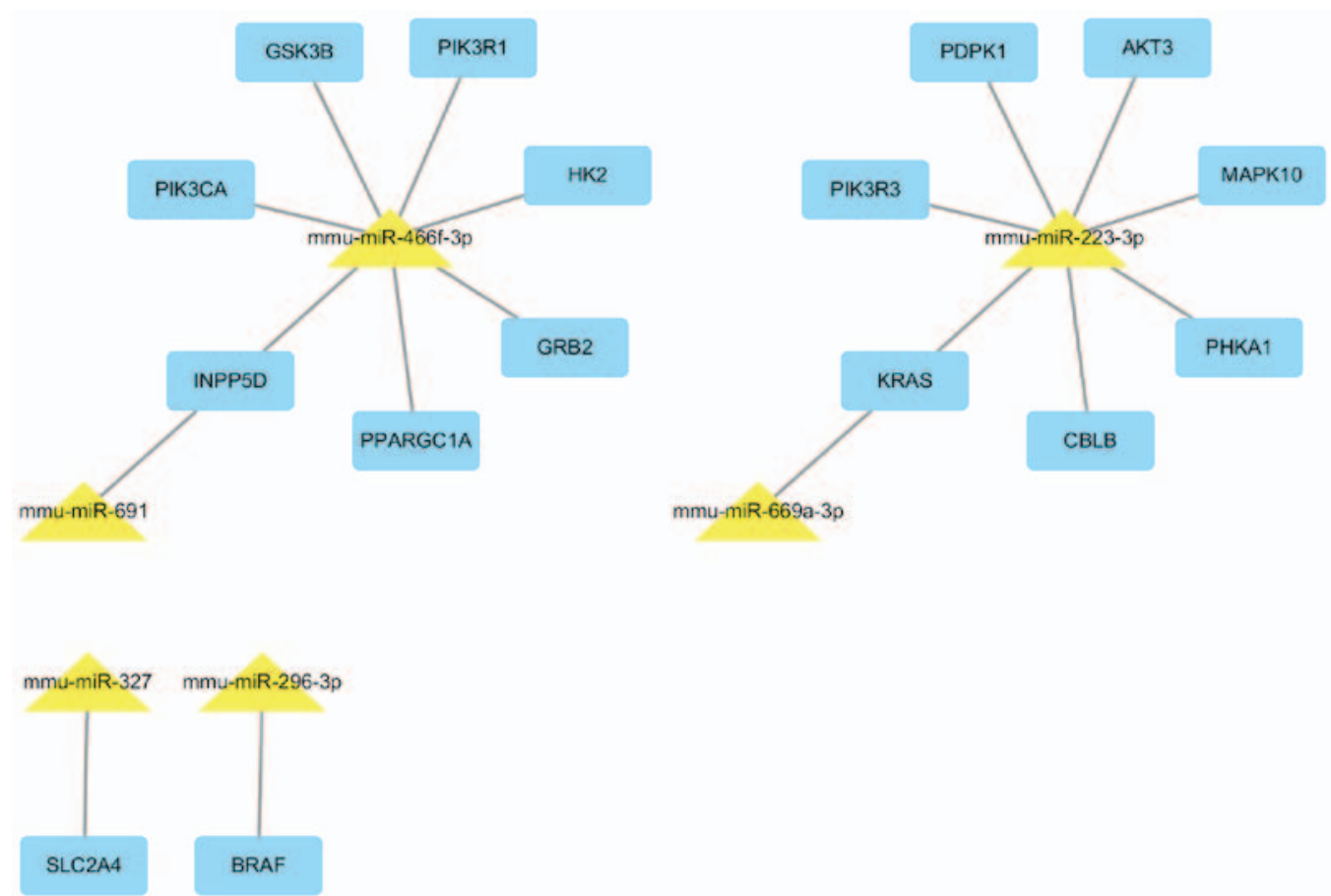

Figure 7. miRNA-gene network contains six miRNAs and their target genes, which are involved in the insulin signaling pathway. Yellow triangular nodes represent miRNA, blue rectangular nodes represent mRNA. Edges describe the inhibitory effect of miRNA on mRNA. miR/miRNA, microRNA.

Previous studies revealed the key role of $\mathrm{Cr}$ in regulating blood glucose $(24,25)$. In addition, Cr supplementation has been reported to improve glucose metabolism in diabetic rats and patients (26-28).
Notably, the present study demonstrated that male offspring from dams fed a LC diet had comparable birth weight to the CON group up to 16 weeks. A maternal LC diet did not change fasting blood glucose levels in offspring at 3 weeks of age; however, 

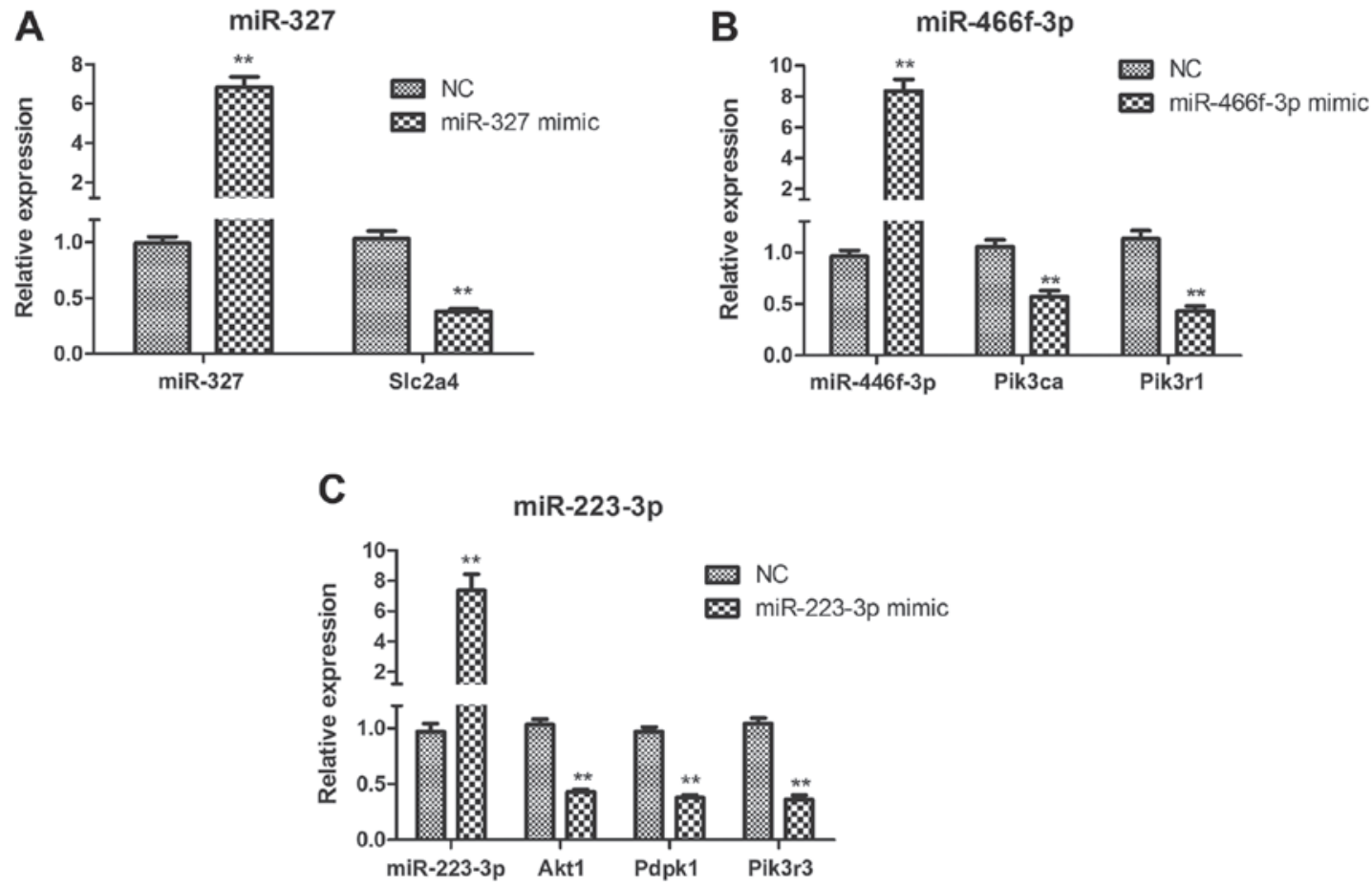

Figure 8. (A-C) Quantitative polymerase chain reaction analysis of miR-327, miR-466f-3p, miR-223-3p, Akt1, Pdpkl, Pik3ca, Pik3rl, Pik3r3 and Slc2a4 expression in miR-327-, miR-466f-3p- and miR-223-3p-transfected HepG2 cells. Data are presented as the means \pm standard deviation, $\mathrm{n}=3$. $^{* *} \mathrm{P}<0.01 \mathrm{vs}$. the NC group. Akt1, thymoma viral proto-oncogene 1; miR/miRNA, microRNA; NC, negative control; Pdpk1, 3-phosphoinositide dependent protein kinase 1; $P i k 3 c a$, phosphatidylinositol 3-kinase, catalytic, $\alpha$ polypeptide; Pik3rl, phosphatidylinositol 3-kinase, regulatory subunit, polypeptide (p85 $\alpha$ ); Pik3r3, phosphatidylinositol 3-kinase, regulatory subunit, polypeptide 3 (p55); Slc2a4, solute carrier family 2 member 4 .

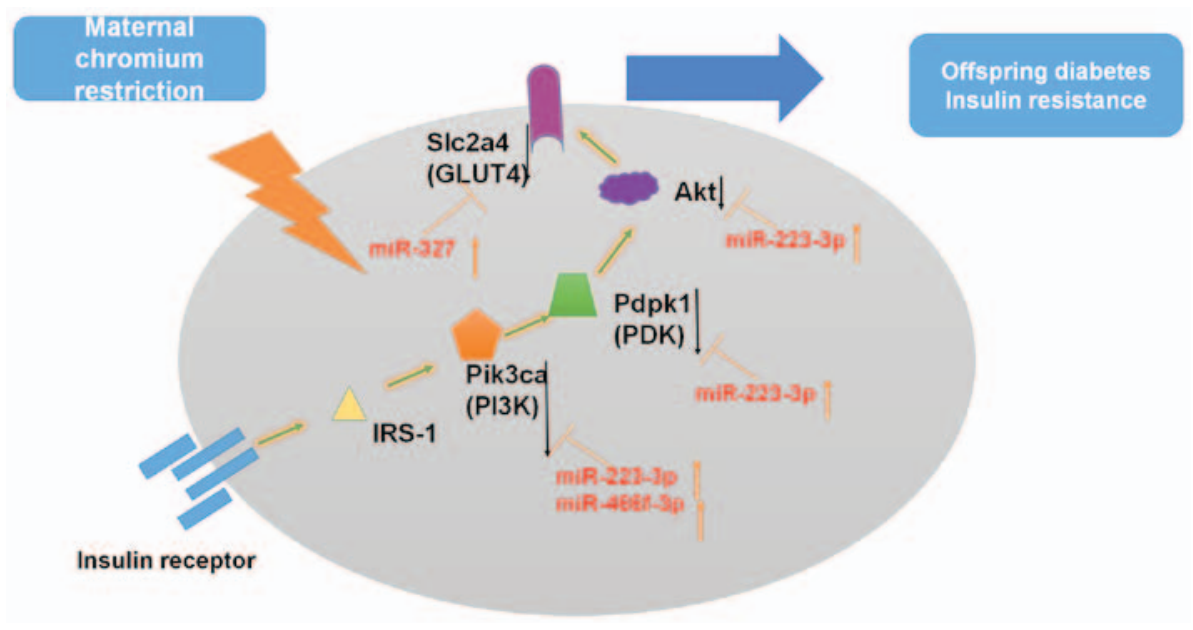

Figure 9. Possible underlying mechanism of miRNA-regulatory networks. Insulin binding to its receptor induces receptor tyrosine autophosphorylation and activation of IRSs/PI3K/Akt signaling pathways. Modulation of gene expression is indicated by black arrows. Akt, protein kinase B; Akt1, thymoma viral proto-oncogene 1; GLUT4, glucose transporter 4; IRS, insulin receptor substrate; miR/miRNA, microRNA; Pdpk1, 3-phosphoinositide dependent protein kinase 1; PI3K, phosphatidylinositol 3-kinase; Pik3ca, phosphatidylinositol 3-kinase, catalytic, $\alpha$ polypeptide; Slc2a4, solute carrier family 2 member 4.

offspring from dams fed a LC diet exhibited impaired fasting blood glucose and glucose tolerance at 16 weeks of age. Mice in the LC/CON group had normal serum Cr levels; however, this could not correct the glucose intolerance. Padmavathi et al indicated that maternal $\mathrm{Cr}$ restriction increased fasting plasma glucose (from 9 months) and the AUC of glucose concentration during OGTT (from 15 months) in the offspring (9). In addition, growth-restricted rats exhibited glucose intolerance from 6 months of age (29). Pups from magnesium-restricted dams, which were shifted to a control diet, exhibited glucose intolerance at 180 days of age (30). A low-protein diet increased fasting blood glucose in offspring at 3 months (31). The results of the present study detected glucose intolerance in offspring earlier than Padmavathi et al (9), this may be partly because the Cr-restricted diet used in the present study was $88 \% \mathrm{Cr}$ restriction compared with $67 \% \mathrm{Cr}$ restriction.

The present study also demonstrated that serum insulin levels and HOMA-IR index were markedly increased in the offspring of dams fed a LC diet at 16 weeks of age. Padmavathi et al reported that maternal $\mathrm{Cr}$ restriction increased fasting insulin (from 
15 months) and HOMA-IR index (from 9 months), whereas rehabilitation could not correct this effect (9). In a previous study, pups from pregnant rats fed a calcium-deficient diet exhibited increased insulin levels and HOMA-IR index at day 200 (32). Furthermore, at 12 months of age, offspring from vitamin B12-restricted dams had higher fasting blood insulin levels and HOMA-IR index (31).

Using a miRNA array and qPCR, the present study indicated that a maternal LC diet downregulated miR-334b-5p expression in the liver of male offspring. Zeng et al revealed that miR-344-3p was downregulated in islets from diabetic GK rats (33). Furthermore, in the present study, a maternal LC diet downregulated miR-296-3p expression in the liver of male offspring. In the pancreatic $\beta$-cell line, MIN6, miR-296 was significantly downregulated by high glucose treatment (34). In addition, a maternal LC diet upregulated miR-122-3p expression in the liver of male offspring; miR-122 is highly expressed in the liver and is highly conserved in sequence (35). Serum miR-122 levels are markedly increased in newly diagnosed diabetic cats (36). A maternal LC diet also upregulated miR-327 expression in the liver of male offspring. miR-327 has been revealed to be upregulated in myocardial microvascular endothelial cells from GK rats compared with Wistar rats (36).

In the present study, further bioinformatics analysis aided in the interpretation of the biological function of the differentially expressed miRNAs in glucose metabolism regulation. According to the KEGG pathway enrichment analysis, the significantly differentially expressed miRNAs (miR-327, miR-223-3p and miR-466f-3p) in the LC/CON group served important roles in the insulin signaling pathway.

In the insulin signaling pathway, Pik3rl and Pik3ca are target genes of miR-466f-3p (37-39). In addition, Aktl and Pik3r3 are target genes of miR-223-3p (40). One target of miR-327 is Slc2a4 (Glut4) (39). In a previous study, Cr malate improved Glut4 and Akt levels in the liver tissues of T2D rats (26). In C2C12 skeletal muscle cells, oligomannuronate $\mathrm{Cr}$ complexes may enhance GLUT4 and the phosphatidylinositol 3-kinase (PI3K)/AKT signaling pathway (25). Therefore, it may be hypothesized that a maternal LC diet upregulates miR-327, miR-466f-3p and miR-223-3p expression, thus inhibiting insulin signaling (Fig. 9).

The PI3K/AKT signaling pathway is a classical pathway that regulates insulin in glucose metabolism. This pathway serves a role in glucose uptake by the liver, skeletal muscles and adipose tissues $(41,42)$. The insulin receptor signaling pathway contributes to the development of diabetes. The IRS-1/PI3K/Akt axis has an important role in the insulin receptor signaling pathway through cascade phosphorylation $(43,44)$. Inhibiting or blocking this pathway may reduce the physiological effects of insulin, which lead to IR. Ye et al demonstrated that hepatocytes in intrauterine growth retardation rats with catch-up growth exhibited decreased IRS1 and PI3K expression (45). In addition, the PI3K p110 $\beta$ subunit, Akt1 proteins and phosphorylated-Akt Ser473 are reduced in the offspring of low protein-fed dams (46). A previous study also reported that maternal protein restriction leads a decrease in Akt1 (Ser473) phosphorylation in the livers of offspring at postnatal day 130 (47). Catch-up growth following intrauterine growth restriction reduces the expression of the PI3K p110 $\beta$ subunit at 22 days old, and Akt phosphorylation and Akt-2 protein levels at 3 months in adipose tissue (48). In addition, maternal diabetes has been revealed to decrease
GLUT4 in the adipose and muscle tissues of 10-week-old male offspring (49).

In conclusion, the present study confirmed that a maternal LC diet caused glucose intolerance and IR in male offspring. The present study is the first, to the best of our knowledge, to demonstrate that a maternal LC diet induces miRNA dysfunction in the liver samples of offspring. In particular, a maternal LC diet may upregulate the expression of miR-327, miR-466f-3p and miR-223-3p, thus inhibiting the insulin signaling pathway in male offspring. In the future, scientists should aim to identify interventions for maternal $\mathrm{Cr}$ restriction to affect glucose metabolism in offspring. This treatment will benefit mothers and the next generation.

\section{Acknowledgements}

The present study was supported by the National Natural Science Foundation of China (grant nos. 81170736 and 81570715), the National Natural Science Foundation for Young Scholars of China (grant no. 81300649), the China Scholarship Council foundation (grant no. 201308110443), the PUMC Youth Fund (grant no. 33320140022), the Fundamental Research Funds for the Central Universities, and the Scientific Activities Foundation for Selected Returned Overseas Professionals of Human Resources and Social Security Ministry. The authors would like to thank Beijing Compass Biotechnology Company for technical assistance with the miRNA microarray experiments.

\section{Acknowledgements}

The normalized data and raw 'cel' files from the miRNA array are available at the NCBI GEO website under the series record GSE82026 (http://www.ncbi.nlm.nih.gov/geo/query/acc.cgi?tok en=upsxikamhpgvbmn\&acc=GSE82026; private website only for reviewers).

\section{References}

1. Diabetes Atlas IDF. 7th edition. International Diabetes Federation, 2015.

2. Hales CN, Barker DJ, Clark PM, Cox LJ, Fall C, Osmond C and Winter PD: Fetal and infant growth and impaired glucose tolerance at age 64. BMJ 303: 1019-1022, 1991.

3. Hales CN and Barker DJ: Type 2 (non-insulin-dependent) diabetes mellitus: The thrifty phenotype hypothesis. Diabetologia 35: 595-601, 1992.

4. de Rooij SR, Painter RC, Phillips DI, Osmond C, Michels RP, Godsland IF, Bossuyt PM, Bleker OP and Roseboom TJ: Impaired insulin secretion after prenatal exposure to the Dutch famine. Diabetes Care 29: 1897-1901, 2006.

5. Food and Nutrition Board: Recommended Dietary Allowances. National Research Council, National Academy of Sciences, Washington DC, 2000.

6. Jeejeebhoy KN, Chu RC, Marliss EB, Greenberg GR and Bruce-Robertson A: Chromium deficiency, glucose intolerance, and neuropathy reversed by chromium supplementation, in a patient receiving long-term total parenteral nutrition. Am J Clin Nutr 30: 531-538, 1977.

7. Anderson RA: Chromium, glucose intolerance and diabetes. J Am Coll Nutr 17: 548-555, 1998.

8. Freund H, Atamian S and Fischer JE: Chromium deficiency during total parenteral nutrition. JAMA 241: 496-498, 1979.

9. Padmavathi IJ, Rao KR and Raghunath M: Impact of maternal chromium restriction on glucose tolerance, plasma insulin and oxidative stress in WNIN rat offspring. J Mol Endocrinol 47: 261-271, 2011. 
10. Waterland RA and Jirtle RL: Early nutrition, epigenetic changes at transposons and imprinted genes, and enhanced susceptibility to adult chronic diseases. Nutrition 20: 63-68, 2004.

11. Zhang Q, Xiao XH, Zheng J, Li M, Yu M, Ping F, Wang Z, Qi C, Wang $T$ and Wang $X$ : Maternal chromium restriction modulates miRNA profiles related to lipid metabolism disorder in mice offspring. Exp Biol Med (Maywood) 242: 1444-1452, 2017.

12. Herrera BM, Lockstone HE, Taylor JM, Wills QF, Kaisaki PJ, Barrett A, Camps C, Fernandez C, Ragoussis J, Gauguier D, et al: MicroRNA-125a is over-expressed in insulin target tissues in a spontaneous rat model of Type 2 Diabetes. BMC Med Genomics 2: $54,2009$.

13. Jordan SD, Krüger M, Willmes DM, Redemann N, Wunderlich FT, Brönneke HS, Merkwirth C, Kashkar H, Olkkonen VM, Böttger T, et al: Obesity-induced overexpression of miRNA-143 inhibits insulin-stimulated AKT activation and impairs glucose metabolism. Nat Cell Biol 13: 434-446, 2011.

14. Zhou B, Li C, Qi W, Zhang Y, Zhang F, Wu JX, Hu YN, Wu DM, Liu Y, Yan TT, et al: Downregulation of miR-181a upregulates sirtuin-1 (SIRT1) and improves hepatic insulin sensitivity. Diabetologia 55: 2032-2043, 2012.

15. Ryu HS, Park SY, Ma D, Zhang J and Lee W: The induction of microRNA targeting IRS-1 is involved in the development of insulin resistance under conditions of mitochondrial dysfunction in hepatocytes. PLoS One 6: e17343, 2011.

16. Jeong HJ, Park SY, Yang WM and Lee W: The induction of miR-96 by mitochondrial dysfunction causes impaired glycogen synthesis through translational repression of IRS-1 in SK-Hep1 cells. Biochem Biophys Res Commun 434: 503-508, 2013.

17. Dou L, Zhao T, Wang L, Huang X, Jiao J, Gao D, Zhang H, Shen T, Man Y, Wang S, et al: miR-200s contribute to interleukin-6 (IL-6)-induced insulin resistance in hepatocytes. J Biol Chem 288: 22596-22606, 2013.

18. National Research Council Committee for the Update of the Guide for the $\mathrm{C}$ and Use of Laboratory A: The National Academies Collection: Reports funded by National Institutes of Health. Guide for the Care and Use of Laboratory Animals. 8th (ed). National Academies Press (US) National Academy of Sciences. Washington (DC), 2011.

19. Yokomizo H, Inoguchi T, Sonoda N, Sakaki Y, Maeda Y, Inoue T, Hirata E, Takei R, Ikeda N, Fujii M, et al: Maternal high-fat diet induces insulin resistance and deterioration of pancreatic $\beta$-cell function in adult offspring with sex differences in mice. Am J Physiol Endocrinol Metab 306: E1163-E1175, 2014.

20. Chou CH, Chang NW, Shrestha S, Hsu SD, Lin YL, Lee WH, Yang CD, Hong HC, Wei TY, Tu SJ, et al: miRTarBase 2016 Updates to the experimentally validated miRNA-target interactions database. Nucleic Acids Res 44: D239-D247, 2016.

21. Livak KJ and Schmittgen TD: Analysis of relative gene expression data using real-time quantitative PCR and the 2(-Delta Delta C(T)) Method. Methods 25: 402-408, 2001

22. Dennis G Jr, Sherman BT, Hosack DA, Yang J, Gao W, Lane HC and Lempicki RA: DAVID: Database for annotation, visualization, and integrated discovery. Genome Biol 4: 3, 2003

23. Shannon P, Markiel A, Ozier O, Baliga NS, Wang JT, Ramage D, Amin N, Schwikowski B and Ideker T: Cytoscape: A software environment for integrated models of biomolecular interaction networks. Genome Res 13: 2498-2504, 2003.

24. Król E and Krejpcio Z: Chromium(III) propionate complex supplementation improves carbohydrate metabolism in insulin-resistance rat model. Food Chem Toxicol 48: 2791-2796, 2010.

25. Hao C, Hao J, Wang W, Han Z, Li G, Zhang L, Zhao X and Yu G: Insulin sensitizing effects of oligomannuronate-chromium (III) complexes in C2C12 skeletal muscle cells. PLoS One 6: e24598, 2011.

26. Feng W, Zhao T, Mao G, Wang W, Feng Y, Li F, Zheng D, Wu H, Jin D, Yang L, et al: Type 2 diabetic rats on diet supplemented with chromium malate show improved glycometabolism, glycometabolism-related enzyme levels and lipid metabolism. PLoS One 10: e0125952, 2015.

27. Feng W, Mao G, Li Q, Wang W, Chen Y,Zhao T, Li F, Zou Y, Wu H, Yang L, et al: Effects of chromium malate on glycometabolism, glycometabolism-related enzyme levels and lipid metabolism in type 2 diabetic rats: A dose-response and curative effects study. J Diabetes Investig 6: 396-407, 2015

28. Racek J, Sindberg CD, Moesgaard S, Mainz J, Fabry J, Müller L and Rácová K: Effect of chromium-enriched yeast on fasting plasma glucose, glycated haemoglobin and serum lipid levels in patients with type 2 diabetes mellitus treated with insulin. Biol Trace Elem Res 155: 1-4, 2013.

29. Intapad S, Dasinger JH, Brown AD, Fahling JM, Esters J and Alexander BT: Glucose intolerance develops prior to increased adiposity and accelerated cessation of estrous cyclicity in female growth-restricted rats. Pediatr Res 79: 962-970, 2016.
30. Venu L, Kishore YD and Raghunath M: Maternal and perinatal magnesium restriction predisposes rat pups to insulin resistance and glucose intolerance. J Nutr 135: 1353-1358, 2005.

31. Bringhenti I, Schultz A, Rachid T, Bomfim MA, Mandarimde-Lacerda CA and Aguila MB: An early fish oil-enriched diet reverses biochemical, liver and adipose tissue alterations in male offspring from maternal protein restriction in mice. J Nutr Biochem 22: 1009-1014, 2011.

32. Takaya J, Yamanouchi S and Kaneko K: A calcium-deficient diet in rat dams during gestation and nursing affects hepatic $11 \beta$-hydroxysteroid dehydrogenase- 1 expression in the offspring. PLoS One 9: e84125, 2014.

33. Zeng LQ, Wei SB, Sun YM, Qin WY, Cheng J, Mitchelson K and Xie L: Systematic profiling of mRNA and miRNA expression in the pancreatic islets of spontaneously diabetic Goto-Kakizaki rats. Mol Med Rep 11: 67-74, 2015.

34. Tang X, Muniappan L, Tang G and Ozcan S: Identification of glucose-regulated miRNAs from pancreatic $\{$ beta\} cells reveals a role for miR-30d in insulin transcription. RNA 15: 287-293, 2009.

35. Tsai WC, Hsu SD, Hsu CS, Lai TC, Chen SJ, Shen R, Huang Y, Chen $\mathrm{HC}$, Lee $\mathrm{CH}$, Tsai TF, et al: MicroRNA-122 plays a critical role in liver homeostasis and hepatocarcinogenesis. J Clin Invest 122: 2884-2897, 2012.

36. Fleischhacker SN, Bauersachs S, Wehner A, Hartmann K and Weber K: Differential expression of circulating microRNAs in diabetic and healthy lean cats. Vet J 197: 688-693, 2013.

37. Zhang X, Zuo X, Yang B, Li Z, Xue Y, Zhou Y, Huang J, Zhao X, Zhou J, Yan Y, et al: MicroRNA directly enhances mitochondrial translation during muscle differentiation. Cell 158: 607-619, 2014.

38. Loeb GB, Khan AA, Canner D, Hiatt JB, Shendure J, Darnell RB Leslie CS and Rudensky AY: Transcriptome-wide miR-155 binding map reveals widespread noncanonical microRNA targeting. Mol Cell 48: 760-770, 2012.

39. Leung AK, Young AG, Bhutkar A, Zheng GX, Bosson AD, Nielsen CB and Sharp PA: Genome-wide identification of Ago2 binding sites from mouse embryonic stem cells with and without mature microRNAs. Nat Struct Mol Biol 18: 237-244, 2011

40. Chi SW, Zang JB, Mele A and Darnell RB: Argonaute HITS-CLIP decodes microRNA-mRNA interaction maps. Nature 460: 479-486, 2009.

41. Cantley LC: The phosphoinositide 3-kinase pathway. Science 296: 1655-1657, 2002

42. Cusi K, Maezono K, Osman A, Pendergrass M, Patti ME, Pratipanawatr T, DeFronzo RA, Kahn CR and Mandarino LJ: Insulin resistance differentially affects the PI 3-kinase- and MAP kinase-mediated signaling in human muscle. J Clin Invest 105: 311-320, 2000.

43. Aguirre V, Werner ED, Giraud J, Lee YH, Shoelson SE and White MF: Phosphorylation of Ser307 in insulin receptor substrate-1 blocks interactions with the insulin receptor and inhibits insulin action. J Biol Chem 277: 1531-1537, 2002.

44. Yamauchi T, Kaburagi Y, Ueki K, Tsuji Y, Stark GR, Kerr IM, Tsushima T, Akanuma Y, Komuro I, Tobe K, et al: Growth hormone and prolactin stimulate tyrosine phosphorylation of insulin receptor substrate- $1,-2$, and -3 , their association with p85 phosphatidylinositol 3-kinase (PI3-kinase), and concomitantly PI3-kinase activation via JAK2 kinase. J Biol Chem 273: 15719-15726, 1998.

45. Ye J,Zheng R, Wang Q, Liao L, Ying Y, Lu H, Cianflone K, Ning Q and Luo X: Downregulating SOCS3 with siRNA ameliorates insulin signaling and glucose metabolism in hepatocytes of IUGR rats with catch-up growth. Pediatr Res 72: 550-559, 2012

46. Martin-Gronert MS, Fernandez-Twinn DS, Bushell M, Siddle K and Ozanne SE: Cell-autonomous programming of rat adipose tissue insulin signalling proteins by maternal nutrition. Diabetologia 59: 1266-1275, 2016.

47. Sohi G, Revesz A and Hardy DB: Nutritional mismatch in postnatal life of low birth weight rat offspring leads to increased phosphorylation of hepatic eukaryotic initiation factor $2 \alpha$ in adulthood. Metabolism 62: 1367-1374, 2013.

48. Berends LM, Fernandez-Twinn DS, Martin-Gronert MS, Cripps RL and Ozanne SE: Catch-up growth following intra-uterine growth-restriction programmes an insulin-resistant phenotype in adipose tissue. Int J Obes 37: 1051-1057, 2013.

49. Kamel MA, Helmy MH, Hanafi MY, Mahmoud SA and Abo Elfetooh $\mathrm{H}$ : Impaired peripheral glucose sensing in $\mathrm{F} 1$ offspring of diabetic pregnancy. J Physiol Biochem 70: 685-699, 2014.

50. Reeves PG: Components of the AIN-93 Diets as improvements in the AIN-96A diet. J Nutr 127: 838S-841S, 1997. International (CC BY-NC-ND 4.0) License. 